\title{
On the horseshoe drag of a low-mass planet. I - Migration in isothermal disks
}

\author{
J. Casoli ${ }^{1}$ \\ Laboratoire AIM, CEA/DSM - CNRS - Université Paris Diderot, Irfu/Service \\ d'Astrophysique, Bât. 709, CEA/Saclay, 91191 Gif-sur-Yvette, France \\ jules.casoli@cea.fr \\ and \\ F. S. Masset ${ }^{2}$ \\ Laboratoire AIM, CEA/DSM - CNRS - Université Paris Diderot, Irfu/Service \\ d'Astrophysique, Bât. 709, CEA/Saclay, 91191 Gif-sur-Yvette, France \\ frederic.masset@cea.fr
}

Received __; accepted _

\footnotetext{
${ }^{1}$ Send offprint requests to jules.casoli@cea.fr

${ }^{2}$ Also at ICF-UNAM, Av. Universidad s/n, Cuernavaca, Morelos, C.P. 62210, México
} 


\begin{abstract}
We investigate the unsaturated horseshoe drag exerted on a low-mass planet by an isothermal gaseous disk. In the globally isothermal case, we use a formalism, based on the use of a Bernoulli invariant, that takes into account pressure effects, and that extends the torque estimate to a region wider than the horseshoe region. We find a result that is strictly identical to the standard horseshoe drag. This shows that the horseshoe drag accounts for the torque of the whole corotation region, and not only of the horseshoe region, thereby deserving to be called corotation torque.

We find that evanescent waves launched downstream of the horseshoe U-turns by the perturbations of vortensity exert a feed-back on the upstream region, that render the horseshoe region asymmetric. This asymmetry scales with the vortensity gradient and with the disk's aspect ratio. It does not depend on the planetary mass, and it does not have any impact on the horseshoe drag. Since the horseshoe drag has a steep dependence on the width of the horseshoe region, we provide an adequate definition of the width that needs to be used in horseshoe drag estimates.

We then consider the case of locally isothermal disks, in which the temperature is constant in time but depends on the distance to the star. The horseshoe drag appears to be different from the case of a globally isothermal disk. The difference, which is due to the driving of vortensity in the vicinity of the planet, is intimately linked to the topology of the flow. We provide a descriptive interpretation of these effects, as well as a crude estimate of the dependency of the excess on the temperature gradient.
\end{abstract}

Subject headings: Planetary systems: formation — planetary systems: protoplanetary 
disks - Accretion, accretion disks - Methods: numerical — Hydrodynamics 


\section{Introduction}

Planetary migration has emerged in the last decades as one of the key processes that shape forming planetary systems. Since the early work of Goldreich \& Tremaine (1979, 1980), who gave the expressions of the tidal torque between a disk and a perturber at an isolated resonance, the tidal torque between a protoplanet and the gaseous disk has been investigated in great detail. In particular, Ward (1986, 1988) worked out the sum of the torques at all Lindblad resonances (the so-called differential Lindblad torque) and found that forming planets should undergo a fast decay towards their central object. Apart from the differential Lindblad torque, the total tidal torque between a planet and a disk features another component, the corotation torque, which arises from material located in the vicinity of the orbit. Most of the early works on planetary migration focused on the Lindblad torque, as it was considered that the latter dominates the corotation torque, and therefore dictates the direction of the migration and the order of magnitude of the drift rate.

Recent works have nevertheless shown the importance of the corotation region on the total torque exerted by the disk on the planet. Traditionally, it was estimated, for low mass planets, by the use of a linear theory. This amounts to evaluating the amplitude of the resonant waves at different azimuthal wave-numbers excited by the planet in the corotation region, and their feed back on the planet. Such an approach was adopted by numerous authors, such as Ward (1989), Korycanskv \& Pollack (1993), and, more recently, by Tanaka et al. (2002). Recently, however, Paardekooper \& Papaloizou (2009b) have stressed the fact that the corotation torque acting on a low mass planet is not correctly accounted for by a linear estimate, except at early stages after a planet is "turned on" in a disk. After a relatively short time scale, the linear estimates break down, and these authors show that the total torque seems to be better described by the sum of the differential Lindblad torque and of the so-called horseshoe drag. This component of the torque arises from particles that 
are close to the corotation and undergo a U-turn in front of or behind the planet. By doing this, they exchange angular momentum with the planet, exerting a torque on it. As the region in which these particles librate is called the horseshoe region, the resulting torque was called the horseshoe drag. It is evaluated by budgeting the jumps of angular momentum of the particles undergoing a U-turn. This was first studied by Ward (1991), and more recently by Masset (2001, 2002); Masset \& Papaloizou (2003); Paardekooper \& Papaloizou $(2009 \mathrm{~b})$. This raises the question of the distribution of the torque density in the coorbital region. While a linear analysis shows that the corotation torque arises from an annulus of radial width $\sim H$ around corotation, the horseshoe drag approach considers only the horseshoe region, which can be arbitrarily thin, and whose width formally tends to zero as the planetary mass does. One can therefore wonder if there is a torque exerted by the material close to the orbit, but outside of the horseshoe region, and more generally how to connect the linear analysis, which considers the launch of evanescent waves in the coorbital region, and the horseshoe drag, which does not contemplate them. Also, since the coorbital region and horseshoe region become of increasing importance in studies of planetary migration, it is useful to derive a number of properties of this region.

With this in mind, we present in this paper I an approach, valid in a globally isothermal disk, in which we use a Bernoulli invariant to handle the streamlines of the flow both in the horseshoe region and outside of it, and in which we take into account the evanescent pressure waves that arise on the downstream sides of the horseshoe region, and which can act back on the upstream regions. We show that taking these waves into account does not modify the horseshoe drag estimate, but that they induce a rear-front asymmetry of the horseshoe region, that scales with the vortensity gradient. We also work out the surface density response in the corotation region, and we indicate a proper way of estimating the horseshoe region width. This is critical for horseshoe drag estimates based on streamline analysis, since this drag scales with the fourth power of the horseshoe width. Note that 
in paper II, we present a derivation of the horseshoe drag in an adiabatic disk. We use a different invariant to derive it, but parts of the derivation are very similar to the derivation undertaken in the present paper.

We also propose a basic model of the mechanisms taking place in the locally isothermal case, when the disk's temperature depends on the radius. Namely, there is creation of vortensity which takes place near the planet, and which results in a corotation torque excess that differs from the globally isothermal case, and which depends on the temperature gradient. Much like in the adiabatic case considered in paper II, this torque excess comes from an edge term of the horseshoe drag. Nevertheless, owing to the lack of an invariant

along the streamlines in the locally isothermal case, we only provide a rough estimate of the excess, and discuss the differences between the predictions of this estimate and the results of numerical simulations.

\section{Basics}

\subsection{Problem definition and notation}

The system we study is composed of a central star, of mass $M_{*}$, and, orbiting around it, a gaseous disk, with an embedded low-mass planet, of mass $M_{p}$, which has a fixed circular orbit of radius $a$ and an angular frequency $\Omega_{p}$. We assume the disk to be thin, and we use a 2D cylindrical geometry, averaging or integrating all the quantities over $z$.

We place ourselves in the frame corotating with the planet, in which we assume the flow to have reached a steady state. Nevertheless, we explicitly discard in this work possible saturation effects. This amounts to assuming that the material which arrives at the upstream side of the horseshoe U-turns does so with the vortensity of the unperturbed disk. In practice, this means that we consider the flow after a time longer than the time required 
to complete the horseshoe U-turns, but shorter than half the horseshoe libration time. In a real situation, the horseshoe flow achieves many librations over the disk's lifetime, and the horseshoe drag saturates to a level that depends on the dissipative processes at work in the disk (e.g. Masset 2001). Hereafter we restrict ourselves to the unsaturated horseshoe drag. The saturation properties, both in the isothermal and adiabatic cases, will be considered in a forthcoming work.

The angular frequency of a fluid element in the disk at a distance $r$ of the star is $\Omega(r)$. The corotation radius, $r_{c}$, is the radius where the gas is rotating at the same speed as the planet: $\Omega\left(r_{c}\right)=\Omega_{p}$. The disk is made of an ideal gas, and is globally isothermal: its temperature is constant in time and space. Initially, the surface density is a power law of the radius:

$$
\Sigma=\Sigma_{c}\left(r / r_{c}\right)^{-\sigma}
$$

where $\Sigma_{c}$ is the surface density at corotation.

We denote the height of the disk by $H$, and we use the aspect ratio $h=H / a$. As the disk is globally isothermal, the sound speed $c_{s}$ is constant across it, and the aspect ratio is only a function of $r: h=c_{s} /[a \Omega(r)]$.

We make use of the vortensity $w=\omega / \Sigma$, where $\omega$ is the vertical component of the flow's vorticity: $\omega=\vec{\nabla} \times\left.\vec{v}\right|_{z}$. With our notation, we have, initially: $w=w_{c}\left(r / r_{c}\right)^{-\mathcal{V}}$, with $\mathcal{V}=3 / 2-\sigma$.

The particles feel the gravitational potential $\Phi=\Phi_{*}+\Phi_{p}+\Phi_{i}$, where $\Phi_{*}=-G M_{*} / r$ is the star's potential, $\Phi_{p}$ the potential of the planet, softened to avoid computational problems:

$$
\Phi_{p}=-G M_{p} /\left(r^{2}-2 r a \cos \phi+a^{2}+\epsilon^{2}\right)^{1 / 2}
$$

(where $\epsilon$ is the softening length, typically $1 / 3$ of $H$ ), and where $\Phi_{i}$ is the potential's indirect 
term:

$$
\Phi_{i}=\frac{G M_{p}}{a^{2}} r \cos \phi=q a \Omega_{p}^{2} r \cos \phi
$$

where $q=M_{p} / M_{*}$.

The enthalpy is denoted $\eta$. For a globally isothermal disk of temperature $T_{c}$, we have $\eta=T_{c} \ln \left(\Sigma / \Sigma_{c}\right)$.

We make use of the two Oort's constants, estimated at the planet's location, $A_{p}$ and $B_{p}$. We often use their values for a Keplerian disk, which reads : $A_{p}=-3 \Omega_{p} / 4$ and $B_{p}=\Omega_{p} / 4$. The $p$ subscript is mandatory to distinguish $B_{p}$ from the Bernoulli invariant, a quantity we define in section 3.2 ,

We label the different quadrants of the horseshoe region as represented in figure 1. We denote with a subscript $F(R)$ what is in front (at the rear) of the planet, rotation-wise. In a similar manner, we use a subscript $+(-)$ for the part of the disk that has $r>r_{c}\left(r<r_{c}\right)$. Lastly, the upstream (downstream) part of a horseshoe leg is denoted with a subscript $u$ $(d)$.

\subsection{Basic equations}

The governing equations are the one of a non magnetized, non self-gravitating, ideal isothermal gas, in the corotating frame, so that the radial velocity $v_{r}$ is unchanged with respect to the inertial frame, while the angular velocity is $v_{\phi}=r \Omega-r \Omega_{p}$. With this notation the continuity equation reads:

$$
\partial_{t} \Sigma+\frac{1}{r} \partial_{r}\left(\Sigma r v_{r}\right)+\frac{1}{r} \partial_{\phi}\left(\Sigma v_{\phi}\right)=0,
$$

while the Euler equations read, respectively in the radial and azimuthal directions:

$$
\partial_{t} v_{r}+v_{r} \partial_{r} v_{r}+\frac{v_{\phi}}{r} \partial_{\phi} v_{r}-r \Omega_{p}^{2}-2 \Omega_{p} v_{\phi}-\frac{v_{\phi}^{2}}{r}=-\frac{\partial_{r} P}{\Sigma}-\partial_{r} \Phi,
$$




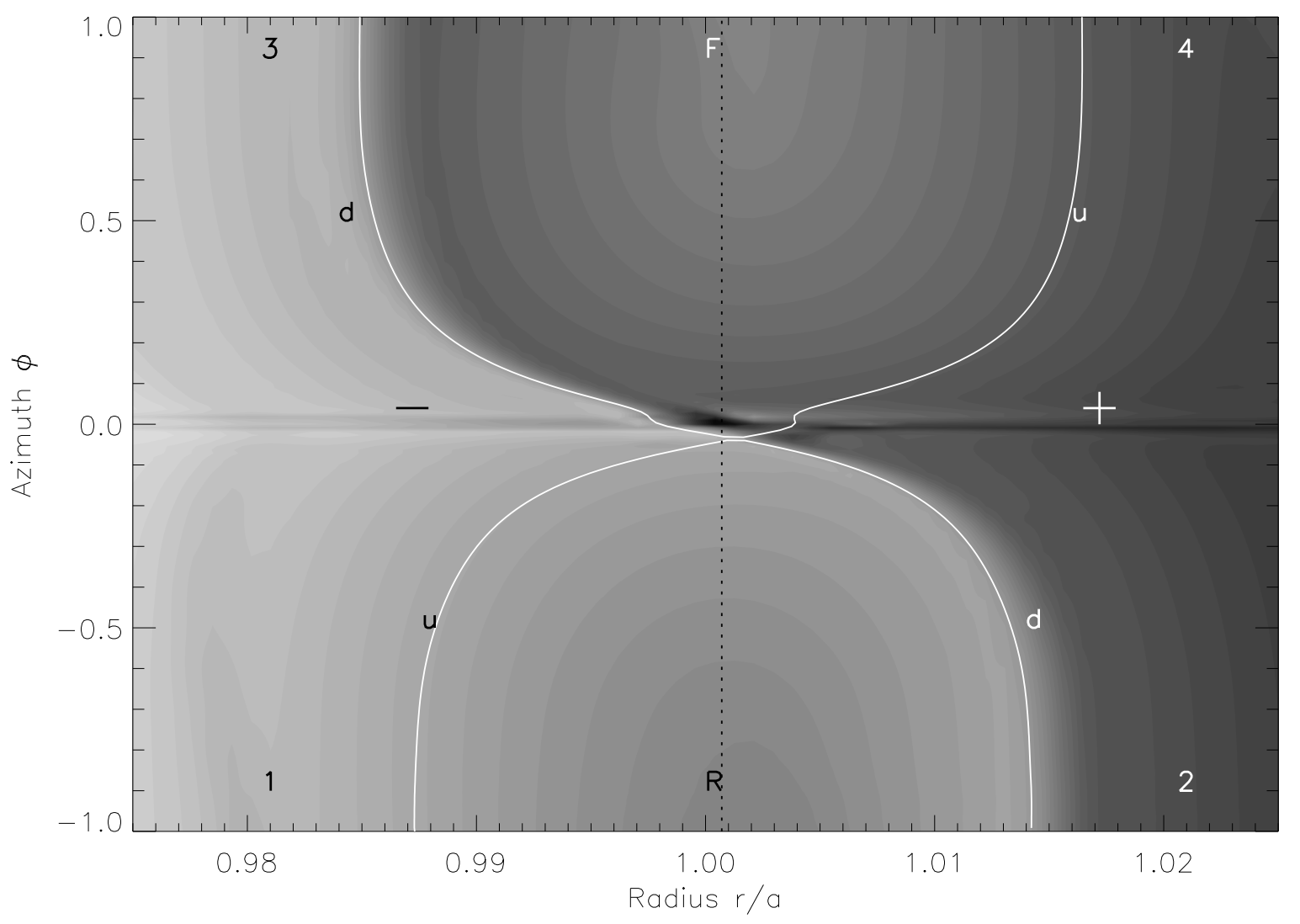

Fig. 1.- An overview of the horseshoe region. This is extracted from the standard simulation (see 4), at $T=25$ orbits. The planet is at $\phi=0$ and $r=1$. The corotation radius is represented by the dotted line. The grey scale represent the total vortensity, and the two white lines are the separatrices. The asymmetry appears clearly, the front part being wider than the rear one. We also show here the integration domains 1 to 4 used in the text. 


$$
\partial_{t} j+v_{r} \partial_{r} j+\frac{v_{\phi}}{r} \partial_{\phi} j=-\frac{\partial_{\phi} P}{\Sigma}-\partial_{\phi} \Phi
$$

where $j=r^{2} \Omega$ is the specific angular momentum.

\section{Horseshoe drag}

\subsection{A first torque expression}

To evaluate the torque exerted on the planet by the disk, we simply budget the angular momentum on a well-chosen domain $D$, located between two azimuths $\phi_{D}$ and $-\phi_{D}$, chosen far enough from the planet to assume that we can neglect $\Phi_{p}$ there. The radial extent of $D$ is bound by two streamlines, which respectively start at $\left(r_{c}+x_{D} ; \phi_{D}\right)$ and $\left(r_{c}-x_{D} ; \phi_{D}\right)$. We assume that $x_{D}$ is much larger than $x_{s}$, the typical half-width of the horseshoe region. The torque exerted by the material inside this region onto the planet is then:

$$
\Gamma_{\text {disk } \rightarrow \text { planet }}=\iint_{D} \Sigma \partial_{\phi} \Phi r d r d \phi
$$

which can be recast, using (6), as:

$$
\Gamma_{\text {disk } \rightarrow \text { planet }}=\iint_{D} \Sigma\left(-\left(\partial_{t} j+v_{r} \partial_{r} j+\frac{v_{\phi}}{r} \partial_{\phi} j\right)-\frac{\partial_{\phi} P}{\Sigma}\right) r d r d \phi
$$

which we note $\Gamma$ from now on. In steady state, we have, using (41):

$$
\begin{aligned}
\Gamma & =\iint_{D} \frac{1}{r}\left(-\partial_{\phi}\left(v_{\phi} \Sigma j\right)-\partial_{r}\left(r \Sigma v_{r} j\right)-\partial_{\phi} r P\right) r d r d \phi \\
& =-\oint_{\partial D} \Sigma j \vec{v} \cdot \overrightarrow{d n}-\oint_{\partial D} r P \overrightarrow{e_{\phi}} \cdot \overrightarrow{d n}
\end{aligned}
$$

where $\overrightarrow{d n}$ is a vector perpendicular to the edge $\partial D$ of the domain $D$, oriented outwards, and with a length equal to the length of the elementary interval of integration on the edge. To proceed in the treatment of this expression, we must rely on the shape of the streamlines. Namely, for $r-r_{c}= \pm x_{D}$, the streamlines are nearly circular and there is therefore no 
advected flux of angular momentum into the domain $D$ through the circular boundaries. We also use the fact that $\overrightarrow{e_{\phi}} \cdot \overrightarrow{d n}=0$ on these boundaries, so that we can ultimately write:

$$
\Gamma=\left[\int_{r_{c}-x_{D}}^{r_{c}+x_{D}}\left[r P+r\left(\Omega-\Omega_{p}\right) \Sigma j\right] d r\right]_{F}^{R},
$$

where the $R$ superscript indicates that the integral has to be performed on the rear side of the domain $D$ (radial boundary at $\phi=-\phi_{D}<0$ ), while the $F$ subscript indicates that the integral has to be performed on the front side of the domain $D$ (radial boundary at $\phi=\phi_{D}>0$ ). In equation (11), the first term of the integrand represents the pressure torque exerted on the material enclosed within the domain $D$, while the second term represents the budget of angular momentum brought to this region by advection. Since the flow is steady in the corotating frame, the angular momentum of this domain is constant in time and the torque is therefore integrally transmitted to the planet. We recognize in the second term of the integrand of equation (11) the classical horseshoe drag expression (Ward 1991, 1992; Masset 2001; Masset \& Papaloizou 2003; Masset et al. 2006a; Paardekooper \& Papaloizou 2009b), but this equation also shows the pressure contribution, which has been overlooked in previous analysis.

\subsection{A Bernoulli invariant}

We derive, from equations (5) and (마) in steady state, a Bernoulli invariant, constant along streamlines in steady state:

$$
B=\frac{v^{2}}{2}+\eta+\Phi-\frac{r^{2} \Omega_{p}^{2}}{2},
$$

where $v^{2}=v_{r}^{2}+v_{\phi}^{2}$.

Far from the planet (i.e. in $\phi_{D}$ and $-\phi_{D}$ ), the streamlines are purely azimuthal, and the fluid elements do not feel the planet's potential, so we get, neglecting the indirect term:

$$
B=\frac{1}{2} r^{2}\left(\Omega-\Omega_{p}\right)^{2}+\phi_{*}(r)-\frac{1}{2} r^{2} \Omega_{p}^{2}+\eta
$$


Following Masset \& Papaloizou (2003), we derive:

$$
\partial_{r} B=r \omega\left(\Omega-\Omega_{p}\right)
$$

where we used the radial equilibrium: $\partial_{r} \phi_{*}=r \Omega^{2}-\partial_{r} \eta$.

Before going further and inject this into equation (11), we evaluate how the Bernoulli invariant is modified at a given location in the disk with respect to its value in the unperturbed disk. Considering a small perturbation $\delta r$ of radius, $\delta \eta$ of enthalpy, and $\delta \Omega$ of angular velocity, the total variation of the Bernoulli invariant is:

$$
\begin{aligned}
\delta B & =r \omega\left(\Omega-\Omega_{p}\right) \delta r-\partial_{r} \eta \delta r+\delta \eta \\
& =\left(\Omega-\Omega_{p}\right) \delta j+\delta^{\prime} \eta
\end{aligned}
$$

where $\delta^{\prime} \eta=\delta \eta-\partial_{r} \eta \delta r$ is the local perturbation of enthalpy, at fixed radius.

\subsection{Final torque expression}

We now evaluate the total torque, using equations (11) and (14), which allows to change the variable of integration to $B$. We use the subscripts (1) to (4) to refer to the quadrants of figure 1, and we get:

$$
\begin{aligned}
\Gamma= & {\left[\int_{r_{c}-x_{D}}^{r_{c}+x_{D}}\left[r P+r\left(\Omega-\Omega_{p}\right) \Sigma j\right] d r\right]_{F}^{R} } \\
= & \int_{B_{\infty}(1)}^{B_{c}} \frac{\Sigma}{\omega} j d B+\int_{B_{c}(2)}^{B_{\infty}} \frac{\Sigma}{\omega} j d B \\
& -\int_{B_{\infty}(3)}^{B_{c}} \frac{\Sigma}{\omega} j d B-\int_{B_{c}(4)}^{B_{\infty}} \frac{\Sigma}{\omega} j d B \\
& +\int_{r_{c}-x_{D}(1)(2)}^{r_{c}+x_{D}} r P d r-\int_{r_{c}-x_{D}(3)(4)}^{r_{c}+x_{D}} r P d r
\end{aligned}
$$

where we supposed the radial boundaries to be far enough in $r_{c} \pm x_{D}$ so we can assume the streamlines to be purely circular there, corresponding to a Bernoulli invariant $B_{\infty}$. 
We note with a subscript 0 the values of the unperturbed flow. The perturbation of angular momentum and pressure are denoted $\delta j$ and $\delta P$, and we use the conservation of vortensity along a horseshoe U-turn in order to write $\Sigma /\left.\omega\right|_{1(4)}=\Sigma /\left.\omega\right|_{2(3)}$. This leads to:

$$
\begin{aligned}
\Gamma= & \left.\int_{B_{\infty}(1)}^{B_{c}} \frac{\Sigma}{\omega}\right|_{1}\left(j_{0}^{-}+\delta j_{1}^{R}\right) d B-\left.\int_{B_{\infty}(2)}^{B_{c}} \frac{\Sigma}{\omega}\right|_{1}\left(j_{0}^{+}+\delta j_{2}^{R}\right) d B \\
& -\left.\int_{B_{\infty}(3)}^{B_{c}} \frac{\Sigma}{\omega}\right|_{4}\left(j_{0}^{-}+\delta j_{3}^{F}\right) d B+\left.\int_{B_{\infty}(4)}^{B_{c}} \frac{\Sigma}{\omega}\right|_{4}\left(j_{0}^{+}+\delta j_{4}^{F}\right) d B \\
& +\int_{r_{c}-x_{D}(1)}^{r_{c}+x_{D}} r\left(P_{0}^{-}+\delta P^{R}\right) d r+\int_{r_{c}-x_{D}(2)}^{r_{c}+x_{D}} r\left(P_{0}^{+}+\delta P^{R}\right) d r \\
& -\int_{r_{c}-x_{D}(3)}^{r_{c}+x_{D}} r\left(P_{0}^{-}+\delta P^{F}\right) d r-\int_{r_{c}-x_{D}(4)}^{r_{c}+x_{D}} r\left(P_{0}^{+}+\delta P^{F}\right) d r
\end{aligned}
$$

The integrals over $r P_{0}^{ \pm}$simplify, between (1) and (3) on the one hand, and (2) and (4) on the other hand. We denote $\Delta j_{0}$ the total jump of angular momentum of a fluid element between the inside and outside region: $\Delta j_{0}(B)=j_{+}^{0}(B)-j_{-}^{0}(B)$, assuming that before and after the jump, the fluid element has the specific angular momentum of the unperturbed flow corresponding to its value of the Bernoulli invariant. By construction $\Delta j_{0}(B)$ is always a positive quantity.

Using equation (16), we obtain:

$$
\delta j^{R}=-\frac{\delta^{\prime} \eta^{R}}{\left(\Omega-\Omega_{p}\right)} .
$$

Since we have $\delta^{\prime} \eta=\delta P / \Sigma$, and $d r=\partial_{B} r d B=d B /\left(r \omega\left(\Omega-\Omega_{p}\right)\right)$, we also can recast the integrals over perturbed pressure using $B$ as an integration variable. We are hence left with:

$$
\begin{aligned}
\Gamma= & -\left.\int_{B_{\infty}}^{B_{c}} \frac{\Sigma}{\omega}\right|_{1} \Delta j_{0}(B) d B-\left.\int_{B_{\infty}(1)}^{B_{c}} \frac{\Sigma}{\omega}\right|_{1} \frac{\delta^{\prime} \eta^{R}}{\left(\Omega-\Omega_{p}\right)} d B+\left.\int_{B_{\infty}(2)}^{B_{c}} \frac{\Sigma}{\omega}\right|_{1} \frac{\delta^{\prime} \eta^{R}}{\left(\Omega-\Omega_{p}\right)} d B \\
& +\left.\int_{B_{\infty}}^{B_{c}} \frac{\Sigma}{\omega}\right|_{4} \Delta j_{0}(B) d B+\left.\int_{B_{\infty}(3)}^{B_{c}} \frac{\Sigma}{\omega}\right|_{4} \frac{\delta^{\prime} \eta^{F}}{\left(\Omega-\Omega_{p}\right)} d B-\left.\int_{B_{\infty}(4)}^{B_{c}} \frac{\Sigma}{\omega}\right|_{4} \frac{\delta^{\prime} \eta^{F}}{\left(\Omega-\Omega_{p}\right)} d B \\
& +\int_{B_{\infty}(1)}^{B_{c}} r \delta^{\prime} \eta^{R} \Sigma \frac{d B}{r \omega\left(\Omega-\Omega_{p}\right)}-\int_{B_{\infty}(2)}^{B_{c}} r \delta^{\prime} \eta^{R} \Sigma \frac{d B}{r \omega\left(\Omega-\Omega_{p}\right)}
\end{aligned}
$$


$-\int_{B_{\infty}(3)}^{B_{c}} r \delta^{\prime} \eta^{F} \Sigma \frac{d B}{r \omega\left(\Omega-\Omega_{p}\right)}+\int_{B_{\infty}(4)}^{B_{c}} r \delta^{\prime} \eta^{F} \Sigma \frac{d B}{r \omega\left(\Omega-\Omega_{p}\right)}$.

If we denote with $B_{s}$ the value of the Bernoulli invariant of the separatrices, we immediately see that $\Delta j_{0}$ is strictly equal to 0 for $B_{\infty}<B<B_{s}$ (i.e. in the region that is not part of the horseshoe region, so that its particles do not undergo any jump), so the integrals over $\Delta j_{0}$ reduces to their values between $B_{s}$ and $B_{c}$. Another simplification arises from the fact that the integrals which were previously over $\delta P$ - i.e. the last four terms of equation (21) - and over $\delta j$ - the second, third, fifth and sixth terms of equation (21) - cancel each other, and we are only left with, reverting the integral boundaries:

$$
\Gamma=\left.\int_{B_{c}}^{B_{s}} \frac{\Sigma}{\omega}\right|_{1} \Delta j_{0}(B) d B-\left.\int_{B_{c}}^{B_{s}} \frac{\Sigma}{\omega}\right|_{1} \Delta j_{0}(B) d B,
$$

which is exactly the original expression derived by Ward (1991). This derivation shows that the whole contribution of the material in the vicinity of corotation, including the material that does not belong to the horseshoe region and circulates beyond the separatrices, reduces to a standard horseshoe drag integral that only involves the vortensity profile inside of the horseshoe region.

\subsection{A model of pressure waves}

Although the torque is of primary interest, it is also worthwhile to have a peek at the anatomy of the horseshoe region, and to stress its major properties. Our first step consists in evaluating the pressure response to the horseshoe dynamics, azimuthally far from the planet.

Before going further, we need to know the response of the disk to any small arbitrary perturbation of vortensity, independent of the azimuth. Indeed, the horseshoe dynamics creates, in the downstream sides of the horseshoe region, stripes of perturbed vortensity, which trigger a density (or pressure) and velocity response. We denote these quantities 
respectively with $\delta \Sigma$ and $\delta v_{\phi}$. We start with the perturbed rotational equilibrium (equation 5]):

$$
-2 \Omega \delta v_{\phi}+c_{s}^{2}\left(\frac{\partial_{x} \delta \Sigma}{\Sigma_{c}}+\frac{\partial_{x} \Sigma_{c}}{\Sigma_{c}} \frac{\delta \Sigma}{\Sigma_{c}}\right)=0,
$$

where the pressure is $P=c_{s}^{2} \Sigma$. Deriving this equation with respect to $x$ leads to:

$$
-2 \Omega_{p} \partial_{x} \delta v_{\phi}+c_{s}^{2} \frac{\partial_{x}^{2} \delta \Sigma}{\Sigma_{c}}=0
$$

where, for a given quantity $\xi$ we neglected all the terms in $\partial_{x} \xi$ comparatively to the ones in $\partial_{x} \delta \xi$, since, as we shall see, the latter scale with $1 / H$, which is much larger than the $1 / r$ scaling of the former. In order to eliminate the unknown $\delta v_{\phi}$ in this equation, we make use of the perturbed vortensity:

$$
\delta w=\frac{\partial_{x} \delta v_{\phi}}{\Sigma_{c}}-\frac{\omega_{0} \delta \Sigma}{\Sigma_{c}^{2}}
$$

so we can write:

$$
\delta \Sigma-\frac{c_{s}^{2}}{\kappa^{2}} \partial_{x}^{2} \delta \Sigma=-\Sigma_{c} \frac{\delta w}{w_{0}}
$$

where $\kappa=\left(2 \Omega_{p} \omega_{c}\right)^{1 / 2}$ is the epicyclic frequency.

The general solution of Eq. (26) is the convolution product of its right hand side by the Green's kernel $K(x)$, which is the solution of:

$$
\delta \Sigma-\frac{c_{s}^{2}}{\kappa^{2}} \partial_{x}^{2} \delta \Sigma=\delta(x)
$$

and whose expression is:

$$
K(x)=\frac{1}{2 H} e^{-|x| / H},
$$

where we have specialized to the Keplerian case, for which $H \equiv c_{s} / \Omega=c_{s} / \kappa$. This kernel represents the pressure response to a singular perturbation of $w$ at $x=0$, of weight $\int w(x) d x=w_{0} / \Sigma_{c}$. We note that $K(x)$ has a unitary weight:

$$
\int_{-\infty}^{+\infty} K(x) d x=1 .
$$


Downstream of the flow, we obtain $\delta w$ straightforwardly by making use of the conservation of vortensity along a streamline, and assuming radially symmetric U-turns (so that fluid particle undergoing a jump at $r=r_{c}+x$ is mapped to $r=r_{c}-x$ ). For the part behind the planet:

$$
\begin{aligned}
\delta w^{R}(x) & =-2 \frac{x}{r_{c}} w_{0} \mathcal{V} \text { for } 0<x<x_{s} \\
& =0 \text { elsewhere. }
\end{aligned}
$$

Similarly, for the part in front of the planet

$$
\begin{aligned}
\delta w^{F}(x) & =-2 \frac{x}{r_{c}} w_{0} \mathcal{V} \text { for } 0>x>-x_{s} \\
& =0 \text { elsewhere. }
\end{aligned}
$$

If we assume $x_{s} \ll H$, this gives for the perturbed surface density (at the rear of the planet, for the sake of definiteness):

$$
\begin{aligned}
\delta \Sigma^{R}(x) & =K * \Sigma_{c} \frac{\delta w}{w_{0}} \\
& =K *\left(-\frac{2 x}{r_{c}} \mathcal{V} \Sigma_{c}\right) \\
& \simeq \int_{0}^{x_{s}} \frac{1}{2 H} \frac{2 x \mathcal{V}}{r_{c}} \Sigma_{c} d x \\
\delta \Sigma^{R}(x) & =\Sigma_{c} \frac{x_{s}^{2} \mathcal{V}}{2 H r_{c}}
\end{aligned}
$$

The equation (33) shows that the actual profile is obtained by convolution of the fictitious surface density profile $\left(-2 x / r_{c}\right) \mathcal{V} \Sigma_{c}$ with the kernel $K$ of unitary weight, which amounts to spreading radially this fictitious profile without changing the total mass of the perturbation. One can check that this fictitious profile is the profile that one would obtain by attributing the vortensity perturbation to the perturbed surface density, without altering the rotation profile (i.e. keeping the profile of vorticity of a Keplerian disk). This helps understanding why the horseshoe drag in a pressure supported disk does not differ from the horseshoe drag estimated by considering test particles (Ward 1991). 
We immediately deduce the corresponding perturbation of enthalpy (which is therefore flat over the horseshoe region because of our hypothesis that $\left.x_{s} \ll H\right)$ :

$$
\delta \eta^{R}=T_{c} \frac{x_{s}^{2} \mathcal{V}}{2 H r_{c}}
$$

Similarly, we have:

$$
\delta \eta^{F}=-T_{c} \frac{x_{s}^{2} \mathcal{V}}{2 H r_{c}}
$$

\subsection{Impact on the horseshoe width}

The value of the Bernoulli invariant at the separatrices is the same as its value at the stagnation point. Quite in contrast with the situation of a non-barotropic disk, for which we shall see in paper II that the value of the invariant is discontinuous at the stagnation point, here the value of the Bernoulli invariant is necessarily continuous in the vicinity of the stagnation point, and the separatrices of the horseshoe region all share the same value $B_{s}$ of the Bernoulli invariant. As a consequence, in order to ensure that it is associated to a Bernoulli invariant with value $B_{s}$, a given separatrix must shift radially, by a value $\delta x_{s}$, in

order to compensate for the variation of the enthalpy due to the evanescent waves. At the rear of the planet, we have, using equations (35) and (16), evaluated at $x=x_{s}$ :

$$
\begin{array}{r}
\delta B=0=4 A_{p} B_{p} x_{s} \delta x_{s}^{R}+T_{c} \frac{x_{s}^{2} \mathcal{V}}{2 H r_{c}} \\
\frac{\delta x_{s}^{R}}{x_{s}}=-\frac{T_{c}}{8 A B r_{c}} \frac{\mathcal{V}}{H}
\end{array}
$$

Or, specializing to the case of a Keplerian disk:

$$
\frac{\delta x_{s}^{R}}{x_{s}}=-\frac{2}{3} \mathcal{V} h .
$$

A similar result holds in front of the planet:

$$
\frac{\delta x_{s}^{F}}{x_{s}}=\frac{2}{3} \mathcal{V} h .
$$


We deduce the estimate of the relative asymmetry of the horseshoe region:

$$
\frac{x_{s}^{F}-x_{s}^{R}}{\left(x_{s}^{F}+x_{s}^{R}\right) / 2}=\frac{4}{3} \mathcal{V} h
$$

The horseshoe region therefore becomes asymmetric when there is a vortensity gradient. The relative asymmetry scales with the vortensity gradient and the disk's aspect ratio, and it is independent of the planetary mass.

\section{Numerical simulations}

We performed simulations of our system using the FARGO code (Masset 2000a,, $\mathrm{b})$, in order to check the properties predicted by the above analysis.

\subsection{Numerical Setup}

All our runs are performed starting with a reference run, and then changing only one parameter. The spatial resolutions of these runs is 700 zones in azimuth and 474 in radius, for a ring spawning radially from $0.8 a$ to $1.2 a$. We stress that, even if the radial width of our mesh seems small, we apply damping conditions at the boundaries that ensure no reflection of the wake. The damping factor was tuned in order to ensure that this assumption is valid.

We use a planet with a mass ratio to the primary of $8 \times 10^{-6}$ (approximately $2.6 M_{\oplus}$, for a central star of mass $\left.1 M_{\odot}\right)$. The initial conditions are presented in section 2.1. For the surface density, we use a typical value of $\sigma=-1$ (this value is not realistic as it corresponds to a surface density increasing outwards, but it corresponds to a large vortensity gradient which exacerbates the effects that we wish to check). The surface density at corotation is $\Sigma_{c}=2 \times 10^{-3}$, and the aspect ratio $h$ is set to 0.05 at $r=r_{c}$.

The last important parameter is the potential softening length. We take it to be $30 \%$ 
of the disk thickness, which is a rather low value. Changing this value can have significant effects, which are discussed in section 6.3.

We indeed observe that the horseshoe region is asymmetric, by comparing its front and rear width. We measure it at $\phi= \pm 1$ rad., far enough not to feel the planet's potential, and once the steady state is reached (after about 25 orbits). As it is taken in steady state, when the separatrix is well defined, we measure the whole full width (hereafter FW) of the separatrix, rather than twice the upstream half-width (distance between the upstream separatrix and the corotation, hereafter TUHW). The difference between the two methods turned out to be about 3-4\%, which is small, but about the same order than the effect we are looking for. In fact, we can see this as an effect of pressure. Here, contrary to our assumptions of section 3.4, we do not strictly have $x_{s} \ll H\left(x_{s} \sim 0.01 a\right)$, so the shape of the pressure wave plays a small role, with a larger amplitude on the downstream part of the U-turn. To get rid of this problem, we chose to use the FW, more appropriate here.

\subsection{Dependence on the vortensity gradient}

Our first step was to conduct simulations with a varying vortensity gradient, in order to quantify the front-rear asymmetry of the horseshoe region. We performed 50 runs with a value of $\mathcal{V}$ varying from -4.5 to 4.5 . The results are presented in figure 2, As we measure the relative asymmetry, the discrepancy between the FW an the TUHW is obvious here, the effect being cumulative (for a positive $\mathcal{V}$, the front $\mathrm{FW}$ is greater than the TUHW, while the rear FW is lower than the TUHW). The fit of the numerical results with our expression is very good. 


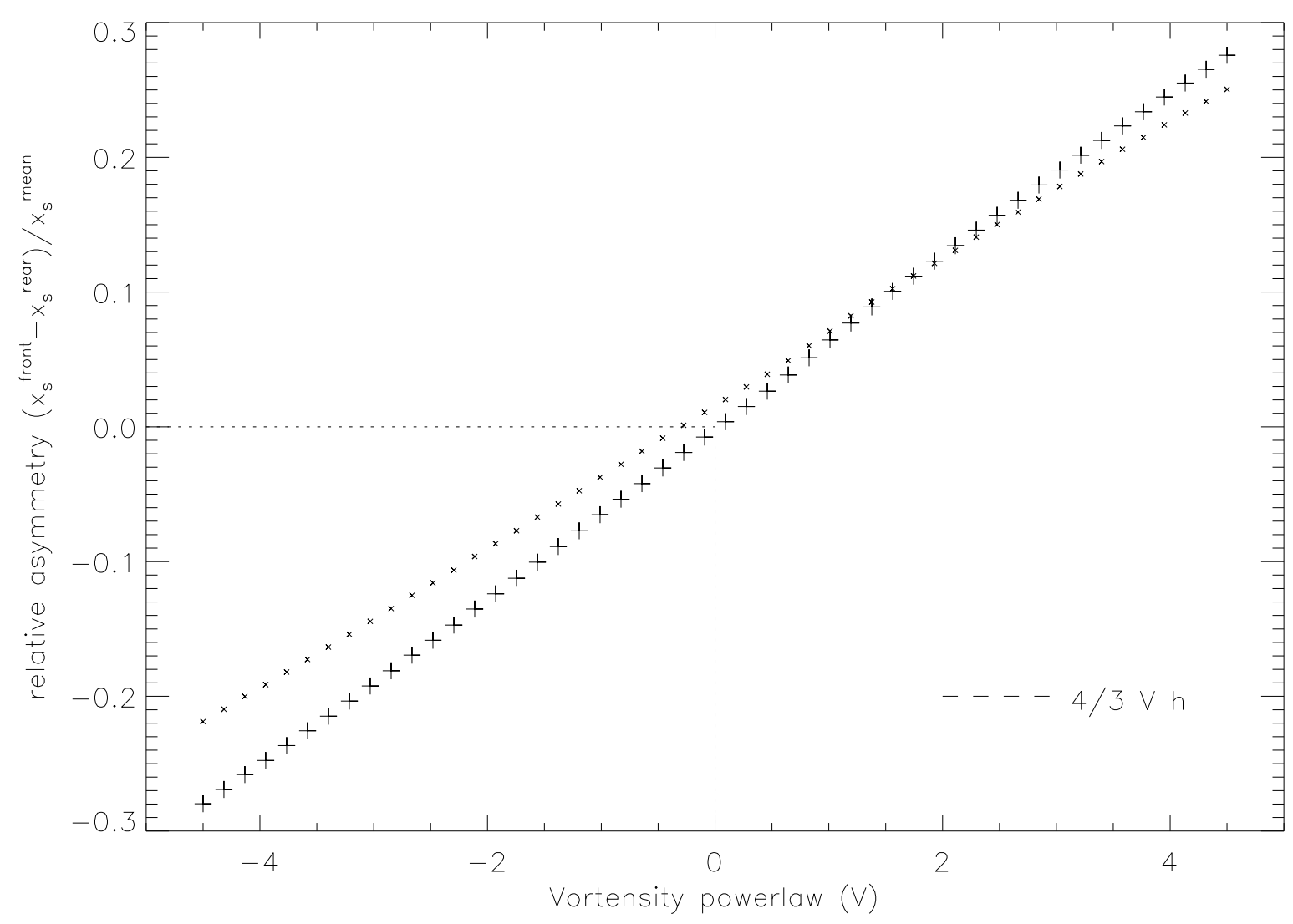

Fig. 2.- Relative asymmetry as a function of the vortensity gradient. The small and large crosses are extracted from simulations, the large crosses representing the asymmetry resulting from the measurement of the FHW, while the small ones correspond to the TUHW (see text). The dashed line represents our analytical expression of equation (41). The fit is very satisfactory, except at very large gradients. Note the relatively high values of the asymmetry: for realistic values of $\mathcal{V}$, the difference in size between the front and rear region can amount to $15 \%$ of the "unperturbed horseshoe width" (which is taken to be the mean of the front and rear widths; see section 5.1). 


\subsection{Dependence on the aspect ratio}

The other factor impacting equation (41) is the aspect ratio. We checked this dependency for some values of $h$, as shown in figure 3. The agreement with the theory is good, but not perfect. It is presumably related to modifications in the topology of the flow, which induce small discrepancies between the runs. This was not a problem for the runs with a varying $\mathcal{V}$, where the streamline topology remained essentially unchanged between the different runs.

\subsection{Pressure profile}

In section 3.4, we considered $x_{s} \ll H$, so that the perturbed surface density, spread over a distance $H$, is uniform across the horseshoe region. As we have seen, this assumption is not always true, and we try here to relax this assumption, modeling the perturbed surface density as a convolution product of the perturbed vortensity by the Green function $K(x)$, properly normalized. We compare this to the perturbed surface density of our simulations in figure 4. We stress that we have used, for $\delta w$, a truncated triangular profile, in order not to take into account the material close to corotation that did not have time to perform a U-turn. Our simulated profile agrees well with the measured profile from the simulations,

both in amplitude and position. The differences on amplitude may be imputed to an uncertainty in $x_{s}$, which is not perfectly defined here, as the situation is not stricto sensu in steady state.

\subsection{Time evolution}

The asymmetry of the horseshoe zone takes some time to establish. When the planet is "turned on" in the disk, the upstream region remains unperturbed for a while. A 


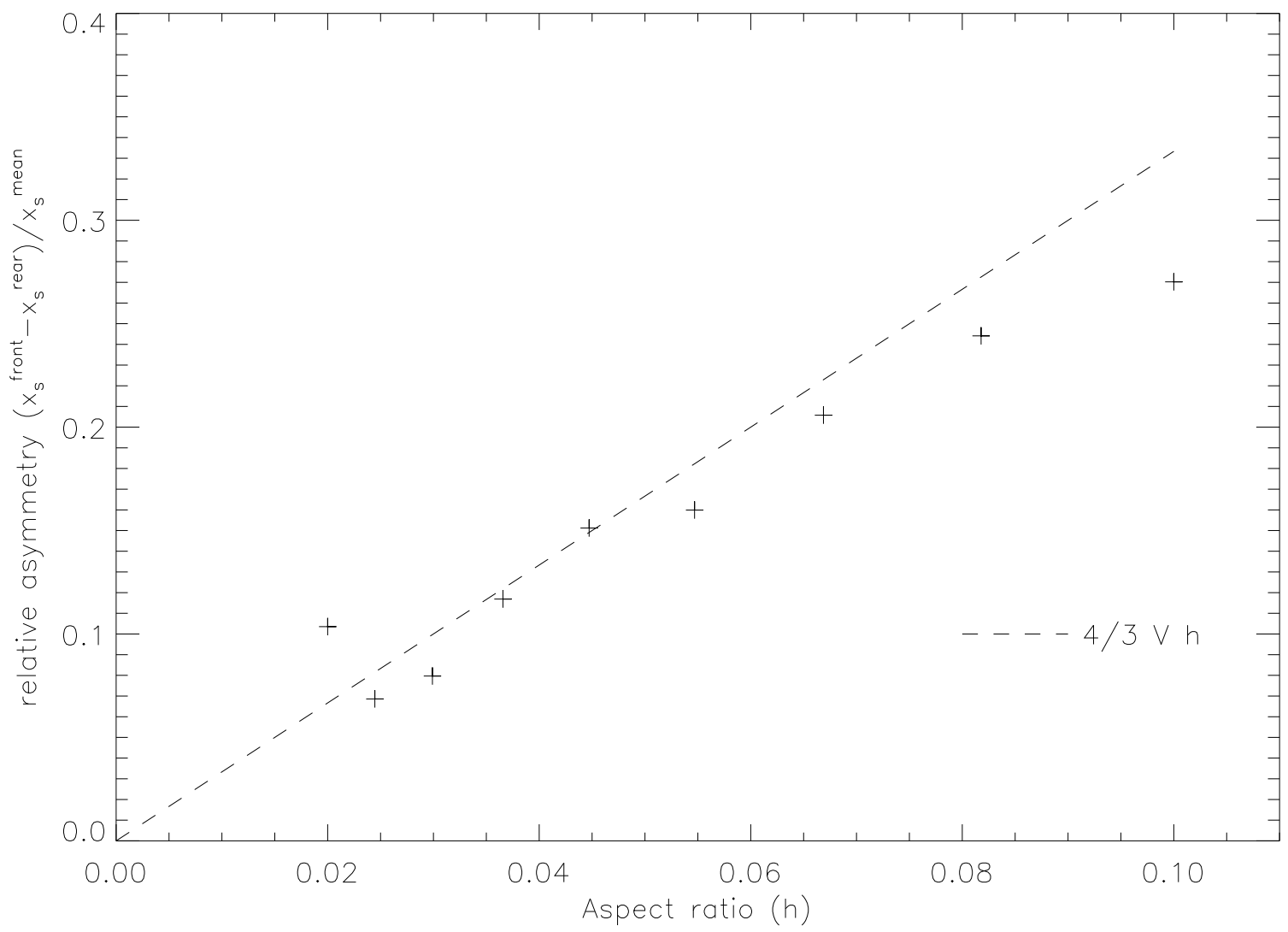

Fig. 3.- Relative asymmetry as a function of the aspect ratio. The dashed line represents our analytical expression. The points are extracted from simulations, which have the standard parameters, except for the aspect ratio. Again, there is a satisfactory agreement, albeit not as good as the dependency on $\mathcal{V}$. 


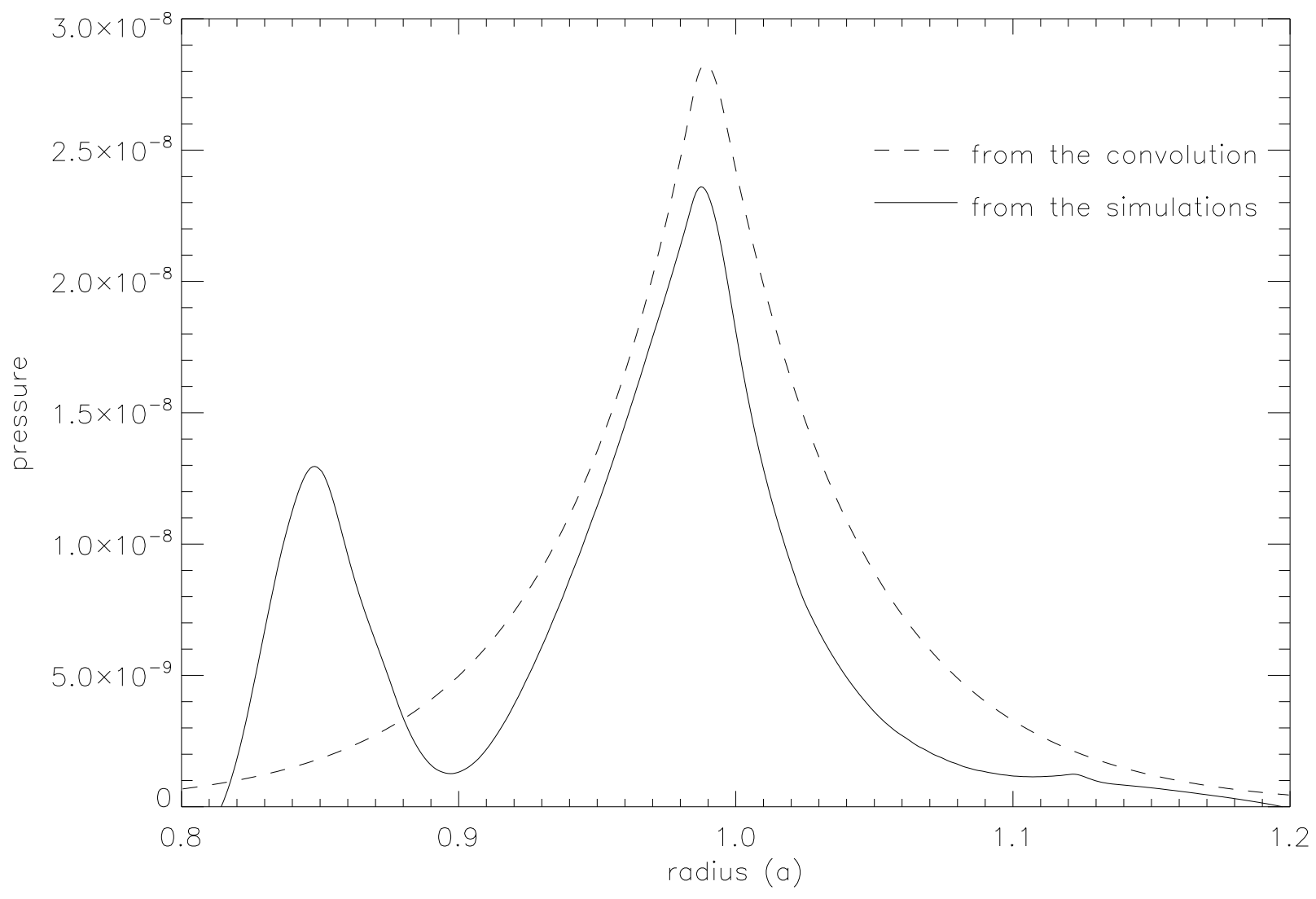

Fig. 4.- Radial pressure profile. This plot is obtained at an azimuth of $\phi=1$, for a run with the standard parameters of section 4.1. We compare the theoretical profile obtained by convolution of the vortensity perturbation by the evanescent waves kernel, properly weighted, as indicated by equation (32), and the outputs from the simulations. The shapes are very similar in the corotation region (our model, which assumes that the response is taken sufficiently far from the planet to be invariant in $\phi$, breaks down in the region of Lindblad resonances because of the presence of the wake). 
typical time for pressure waves to develop is the time needed for particles to perform a horseshoe U-turn. Following Baruteau \& Masset (2008), this time is approximatively: $\tau_{U-t u r n}=\Omega_{p}^{2} h^{3 / 2} q^{-1 / 2} /\left(A_{p}^{2} B_{p}\right)$. This is in good agreement with our simulations, as shown in figure 5. Here, a small initial asymmetry (approx. 1/3 of the final asymmetry) already exists, which we did not investigate, owing to its transient behavior.

\subsection{Impact of the mass}

Since we considered the low-mass planet limit, we want to know to which extent our expression is true. To do so, we performed calculations varying the planet mass to primary mass ratio (see figure [6). We see that our expression stand up to $q \simeq 2 \times 10^{-5}$ (approx. $7 M_{\oplus}$ ), significantly over the usual "low-mass" planet limit (Masset et al. 2006a). Presumably this is because the condition $x_{s}<H$ can be marginally verified while equations (39) and (401) still hold.

\section{Discussion}

We discuss hereafter a number of points related to our results.

\subsection{A definition of the horseshoe width}

The torque expression of equation (22) is the one derived by Ward (1991), except that the integral boundaries are expressed in terms of values of the Bernoulli invariant, rather than $x_{s}$. For practical purposes, one would wish to have an expression in terms of the horseshoe half width. The latter is ill-defined, however, owing to the asymmetry of the horseshoe zone. In order to provide an adequate definition of the average horseshoe width, 


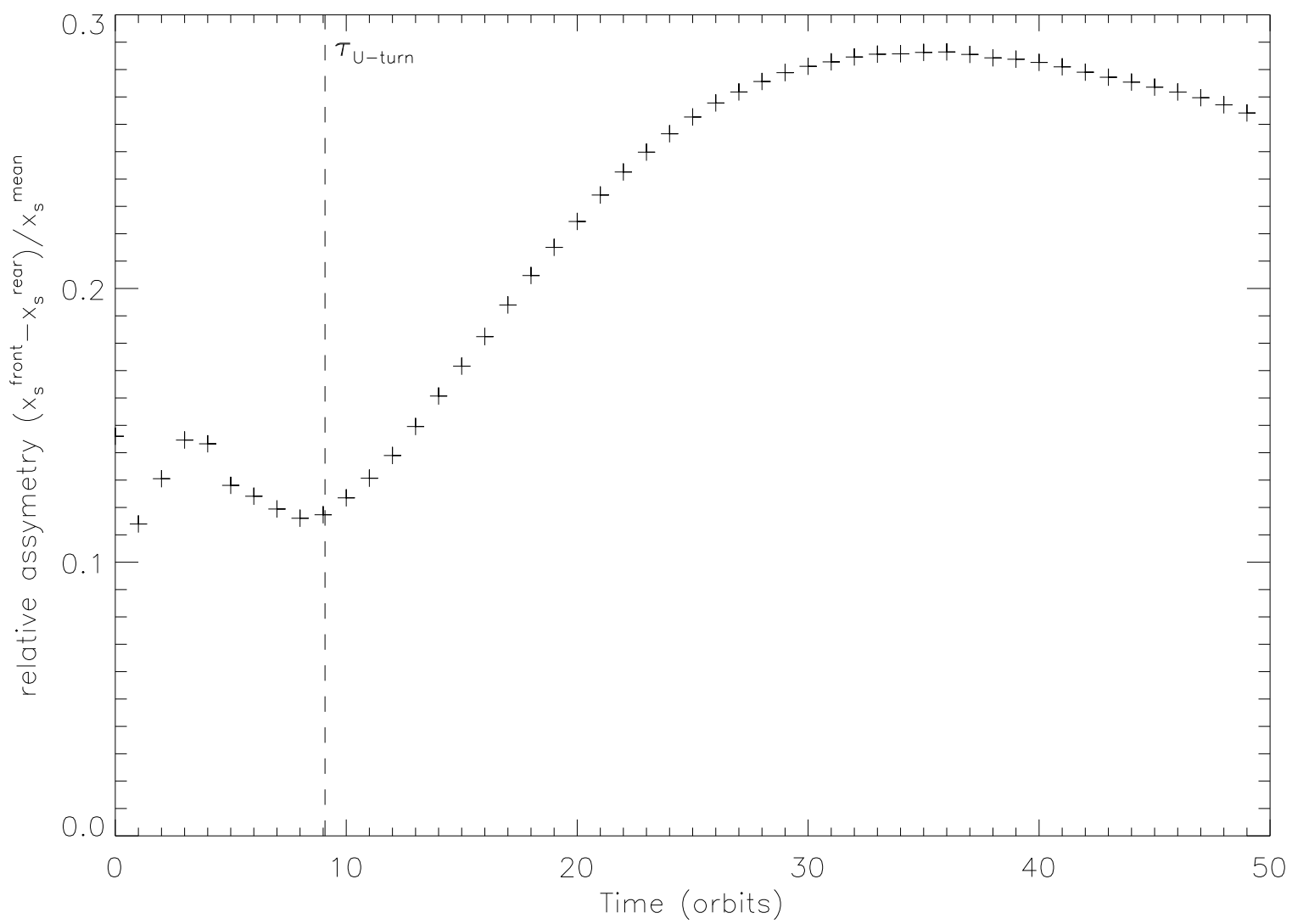

Fig. 5.- Relative asymmetry as a function of time, in orbits. This is a run with standard parameters, except that the aspect ratio is $h=0.08$. The vertical dashed line represent $\tau_{U-\text { turn }}$. Here, the asymmetry boost starts around $\tau_{U-t u r n}$, as expected. Note that the initial asymmetry is not 0 , presumably due to the initial pressure gradient in the disk (disks with vanishing pressure gradients do not exhibit initial asymmetry). 


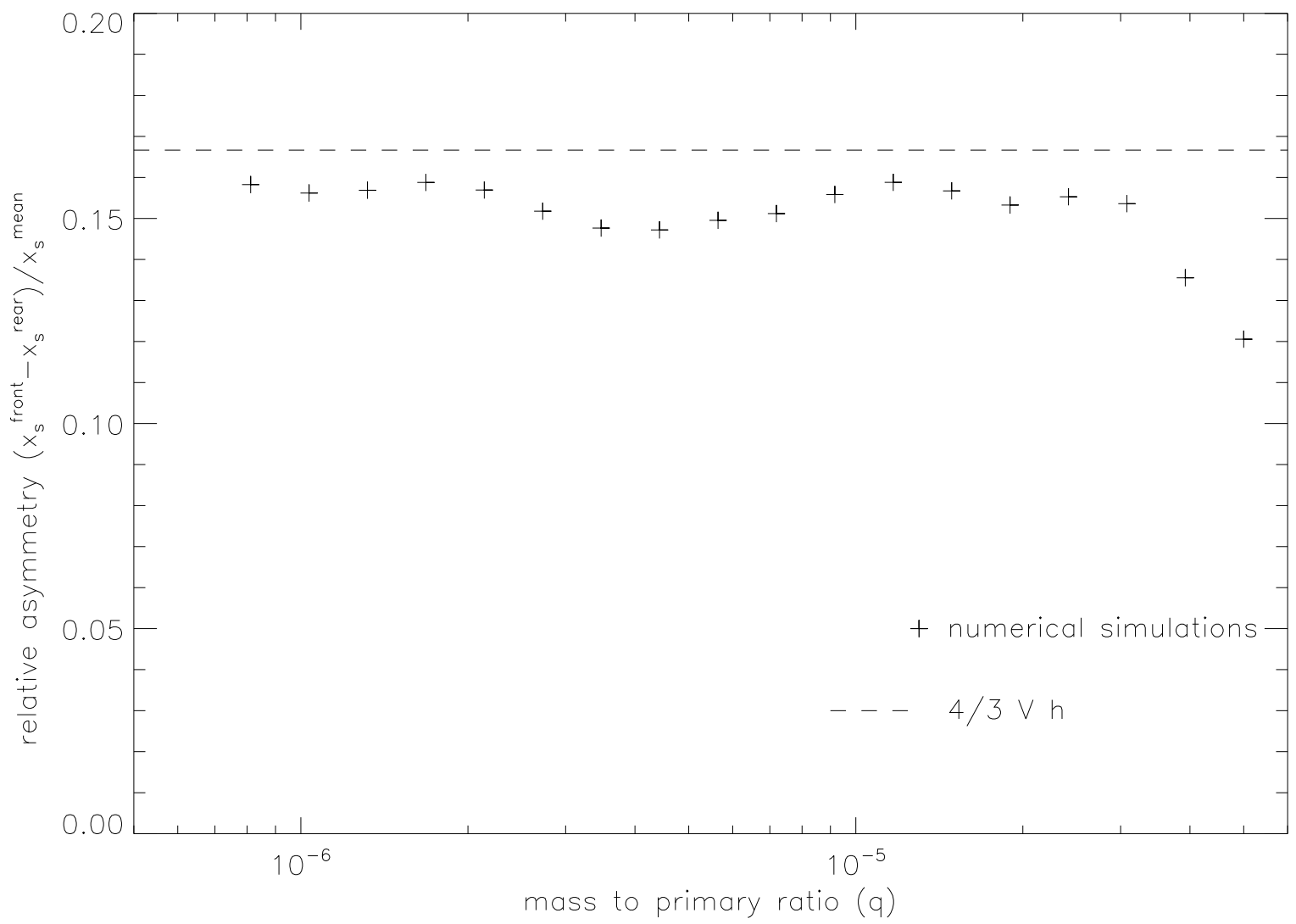

Fig. 6.- Relative asymmetry as a function of the planet mass to primary mass ratio. The dashed line represents our analytical expression, given by equation (41)). The other points are extracted from simulations. Note that each point is measured at a different date, $t_{\text {measure }} \propto 1 / \sqrt{q}$, as the locally steady state takes more time to reach for smaller planets. 
we need to derive $B_{s}$, getting rid of $\delta \eta^{\prime}$. To achieve this, although they share the same value $B_{s}$, we explicitly sum $B_{s}^{F}$ and $B_{s}^{R}$, which yields, to second order in $x$ :

$$
\begin{aligned}
B_{s}^{F}+B_{s}^{R} & =B_{c}+2 A_{p} B_{p}\left(x_{s}^{F}\right)^{2}+\delta^{\prime} \eta^{F}+B_{c}+2 A_{p} B_{p}\left(x_{s}^{R}\right)^{2}+\delta^{\prime} \eta^{R} \\
B_{s} & =B_{c}+2 A_{p} B_{p} \frac{\left(x_{s}^{F}\right)^{2}+\left(x_{s}^{R}\right)^{2}}{2}
\end{aligned}
$$

In order to transform equation (22) so as to get rid of the Bernoulli invariant, we write to the first order in $x$ the factors of the integrand: $\Delta j_{0}(x)=4 B_{p} r_{c} x$ and $\Sigma / \omega=\Sigma_{c} / \omega_{c}\left(1+\mathcal{V} x / r_{c}\right)$. This yields:

$$
\begin{aligned}
\Gamma & =4 B_{p} r_{c} \frac{\Sigma_{c}}{\omega_{c}}\left[\int_{B_{c}}^{B_{s}}|x|\left(1-\mathcal{V} \frac{|x|}{r_{c}}\right) d B-\int_{B_{c}}^{B_{s}}|x|\left(1+\mathcal{V} \frac{|x|}{r_{c}}\right) d B\right] \\
& =-8 B_{p} \frac{\Sigma_{c}}{2 B_{p}} \mathcal{V} \int_{B_{c}}^{B_{s}} \frac{B-B_{c}}{2 A_{p} B_{p}} d B \\
& =\frac{3 \Omega^{2}}{4} \Sigma_{c} \mathcal{V}\left[\frac{\left(x_{s}^{F}\right)^{2}+\left(x_{s}^{R}\right)^{2}}{2}\right]^{2},
\end{aligned}
$$

which is the same torque expression as the one of Ward (1991), provided we take:

$$
x_{s}^{\text {mean }}=\left[\frac{\left(x_{s}^{F}\right)^{2}+\left(x_{s}^{R}\right)^{2}}{2}\right]^{1 / 2}
$$

This gives an adequate definition of the horseshoe width to be used when one wishes to estimate the horseshoe drag by means of a streamline analysis.

\subsection{A direct estimate of the corotation torque}

As we have seen, taking into account the pressure effects does not unveil a different torque value from the one derived by Ward (1991). The main reason that we have mentioned in section 3.4 is the fact that the launch of evanescent waves in the coorbital region renders radially more diffuse the perturbation of surface density (which would otherwise be bound to the horseshoe region), but leaves its linear density unchanged. This result is not surprising, since the fluid model of Goldreich \& Tremaine (1979) had no pressure dependence. 
One can wonder, however, why the horseshoe region asymmetry described in section 3.5 does not have any impact on the torque. In order to gain some insight into the reasons for that, we examine here how this asymmetry alters the front and rear components of the torque by a direct, albeit approximate, estimate of the corotation torque.

The horseshoe U-turns, by conserving the vortensity, produce stripes of perturbed vortensity in front of the planet and behind it, in a disk with a vortensity gradient. These perturbation of vortensity yield perturbations of surface density. Disregarding the radial spread of these perturbations of surface density, we can write their linear mass as:

$$
\Lambda=\int \delta \Sigma(x) d x=\frac{\mathcal{V} x_{s}^{2} \Sigma_{c}}{a}
$$

in front of the planet, and:

$$
\Lambda=-\frac{\mathcal{V} x_{s}^{2} \Sigma_{c}}{a}
$$

behind the planet. We evaluate the torque arising from a stripe of linear mass $\Lambda$ onto the planet, assuming that it starts at a distance $d$ from the latter:

$$
\Gamma_{\text {stripe }} \approx a \int_{d}^{\infty} r_{c} \frac{G M_{p}}{l^{2}} \Lambda d l=\frac{\Omega_{p}^{2} a^{4} q}{d} \Lambda .
$$

We note that the effects of the stripe in front of the planet, and of the stripe that is located behind it, are cumulative, since they involve perturbations of surface density of opposite signs, hence the corotation torque, in total, is:

$$
\Gamma \approx \Omega_{p}^{2} a^{3} q \mathcal{V} \Sigma_{c} \frac{\left(x_{s}^{F}\right)^{2}+\left(x_{s}^{R}\right)^{2}}{d} .
$$

This allows to understand why the horseshoe drag remains unchanged when the horseshoe region becomes asymmetric: $x_{s}^{F}$ increases (decreases) while $x_{s}^{R}$ decreases (increases), but their sum (or the sum of their square) remains constant to lower order in $\mathcal{V}$. Put into simple words, taking for example a positive torque excess $(\mathcal{V}>0)$, the widening of the front region produces a supplementary torque excess, since the front region is producing a 
positive torque excess. But the torque excess produced by the rear region is also positive, so the shrinking of this region induces a smaller torque excess, which balance, to the first order, the supplementary torque excess of the front region, since $\left|\delta x_{s}^{F}\right|=\left|\delta x_{s}^{R}\right|$.

As a side result, we compare equation (48) to the expression of Ward (1991), which reads with our notation, in a Keplerian disk:

$$
\Gamma_{\text {Ward }}=\frac{3}{4} \mathcal{V} \Sigma_{c} x_{s}^{4} \Omega_{p}^{2}
$$

Noting that $x_{s}^{2} \sim a^{2} q / h$ (Masset et al. 2006a; Paardekooper \& Papaloizou 2009a), and assuming that the stripes originate at a distance $\sim H$ from the planet, equation (48) can be recast as:

$$
\Gamma \sim 2 \mathcal{V} \Sigma_{c} x_{s}^{4} \Omega_{p}^{2}
$$

This is the same expression except for a $3 / 8$ factor, presumably originating from our simple estimates of $d$ and $x_{s}$.

\section{Relaxation of the globally isothermal disk hypothesis}

In a globally isothermal disk, the asymmetry of the horseshoe region does not involve additional generation of perturbed surface density, because there is no generation of vortensity, since the gradients of pressure and density are always aligned. However, in a locally isothermal disk, where the temperature depends on the radius $\left(T=T(r)=T_{c}\left(r / r_{c}\right)^{-\tau}\right)$, this is not true anymore, and the vortensity created corresponds to an additional perturbation of surface density that exerts a torque onto the planet. For the sake of definiteness we call this torque the non-isothermal torque excess. For a proper formulation of this torque, one could follow the method of paper II, using an adequate invariant along the streamlines, whenever it exists, in order to derive the downstream vortensity and perturbed surface density. In the present situation, nevertheless, the 
variation of the state variables of a fluid element that goes from infinity to the stagnation point depends on its path, so we cannot use the method of paper II for adiabatic flows. We expect therefore the non-isothermal torque excess to display a strong sensitivity to the topology of the streamlines. As we shall see hereafter, this is indeed the case.

Prior to a fine tuned description of the excess inferred from numerical simulations, we give hereafter an oversimple estimate of the magnitude of the offset, which should apply to situations in which there is only one stagnation point, and which is adapted from a discussion provided in paper II, in which we interpret the adiabatic torque excess as arising from a constitutive asymmetry of the horseshoe region.

\subsection{An oversimple approach}

We know from our simulations that a locally isothermal disk exhibits a non-isothermal torque excess, even for a vanishing vortensity gradient. In order to estimate this excess, we

provide hereafter an estimate of the intrinsic asymmetry of the horseshoe region of a locally isothermal disk. All this study is done for $\mathcal{V}=0$, in order to neglect the asymmetry of the horseshoe region acquired on the long term under the feed back of the evanescent waves, which does not plays a role in the torque, as explained before.

To estimate the intrinsic asymmetry, we consider a fluid element belonging to a separatrix, that starts at $\left(x_{s}, \phi_{\infty}\right)$. By definition the torque applied to it is exactly sufficient to bring it to the stagnation point. For the globally isothermal case, in front of the planet, this reads:

$$
\Delta j_{\mathrm{GI}}=\int_{t_{0}}^{t_{\infty}} \Gamma_{\mathrm{GI}}(t) d t
$$




$$
=\int_{\phi_{\infty}}^{\phi_{s}} \Gamma_{\mathrm{GI}}(\phi)(d t / d \phi) d \phi,
$$

where the GI index stands for "globally isothermal". We then approximate $(d t / d \phi)=$ $1 / v_{\phi} \simeq a / 2 A_{p} x_{s, \mathrm{GI}}$, and since $\Delta j=2 a B_{p} x_{s, \mathrm{GI}}$, we have:

$$
4 A_{p} B_{p} x_{s}^{2}=\int_{\phi_{\infty}}^{\phi_{s}} \Gamma(\phi) d \phi
$$

A similar relation holds in locally isothermal case, with a modified $x_{s}=x_{s, \text { GI }}+\delta x_{s}$ and $\Gamma=\Gamma_{\mathrm{GI}}+\delta \Gamma$. The only contributions in $\delta \Gamma$ is the one of the pressure, assuming that the fluid element follows a similar path in both cases:

$$
\delta \Gamma=\left.\frac{\partial_{\phi} P}{\Sigma}\right|_{\mathrm{LI}}-\left.\frac{\partial_{\phi} P}{\Sigma}\right|_{\mathrm{GI}}=\delta T \frac{\partial_{\phi} \Sigma}{\Sigma},
$$

where the LI index stands for "locally isothermal", and where $\delta T=T_{L I}-T_{G I}$. In equation (54) we have made use of the fact that $\partial_{\phi} T$ cancels out. From equation (53), we have:

$$
8 A_{p} B_{p} \delta x_{s}^{F}=\int_{\phi_{\infty}}^{\phi_{s}} \delta T \frac{\partial_{\phi} \Sigma}{\Sigma} d \phi .
$$

We simplify further by assuming "square", instantaneous U-turns: the particles go from $\left(x_{s}, \phi_{\infty}\right)$ to $\left(x_{s}, \phi_{s}\right)$, then to $\left(-x_{s}, \phi_{s}\right)$, and lastly to $\left(-x_{s}, \phi_{\infty}\right)$. This ensures that $\delta T$ is constant along it, and we are left with:

$$
\begin{aligned}
8 A_{p} B_{p} \delta x_{s}^{F} & \sim \delta T\left(x_{s}\right) \int_{\phi_{\infty}}^{\phi_{s}} \frac{d \Sigma}{\Sigma} \\
& =-\tau \frac{x_{s}}{a} T_{0} \frac{\left(\Sigma_{s}-\Sigma_{\infty}\right)}{\Sigma_{c}}
\end{aligned}
$$

where we have replaced $\delta T$ by its first order expression $-\tau T_{0} x_{s} / a$. Denoting with $\Delta P=P_{s}-P_{\infty}$, this reduces to:

$$
\delta x_{s}^{F}=-\frac{\tau}{8 A_{p} B_{p} a} \frac{\Delta P}{\Sigma_{c}} .
$$

On the rear of the planet, the sign of $\delta T$ changes, and we have $\delta x_{s}^{R}=-\delta x_{s}^{F}$. The crude estimate presented here gives an order of magnitude of the intrinsic asymmetry of the 
horseshoe region, that gives rise to a torque for the reasons presented in detail in paper II. Since the enthalpy tends to fill the planet's potential well, we always have $\Delta P>0$, and the sign of $\delta x_{s}^{F}$ only depends on $\tau$. For example, for a disk with decreasing temperature

$(\tau>0), \delta x_{s}^{F}$ is negative, and the planet feels a negative torque excess from the front region. The rear region being in turn broader, the planet feels a negative torque excess from the rear region also, hence a globally negative torque excess. To quantify this excess, we use the same method as in paper II, which yields:

$$
\delta \Gamma=2 \Sigma_{c} v_{\phi} \delta x_{s} \Delta j \sim 2 \tau \Delta P x_{s}^{2}
$$

where we take $v_{\phi}=2 A_{p} x_{s}$, and $\Delta j=4 B_{p} a x_{s}$.

We therefore find a non-isothermal torque excess that scales with the temperature gradient, and which should have a stronger dependency at lower softening lengths, since the potential well of the planet is then deeper, and $\Delta P$ is therefore larger.

\subsection{Comparison to numerical simulations}

We performed two sets of 30 numerical simulations with a varying temperature slope $\tau$ between -3 and +3 to investigate the behavior of the non-isothermal torque excess. The

first set uses a softening length of $\epsilon=0.3$, while the second one uses $\epsilon=0.5$. Due to a strong generation of vortices when $\mathcal{V}=0$, we adopted a non-vanishing vortensity gradient, and we chose $\mathcal{V}=1$. The results of these simulations are presented in figure 8 , We measured the quantity $\Delta \Gamma$ as explained in figure 7 . The value of $\Delta P$ was measured by averaging $\Delta P^{F}$ and $\Delta P^{R}$.

There is a poor fit of the numerical data with our crude estimate. While the slope has the expected value (within an error range of $\pm 25 \%$ ), there are discontinuities in the case 


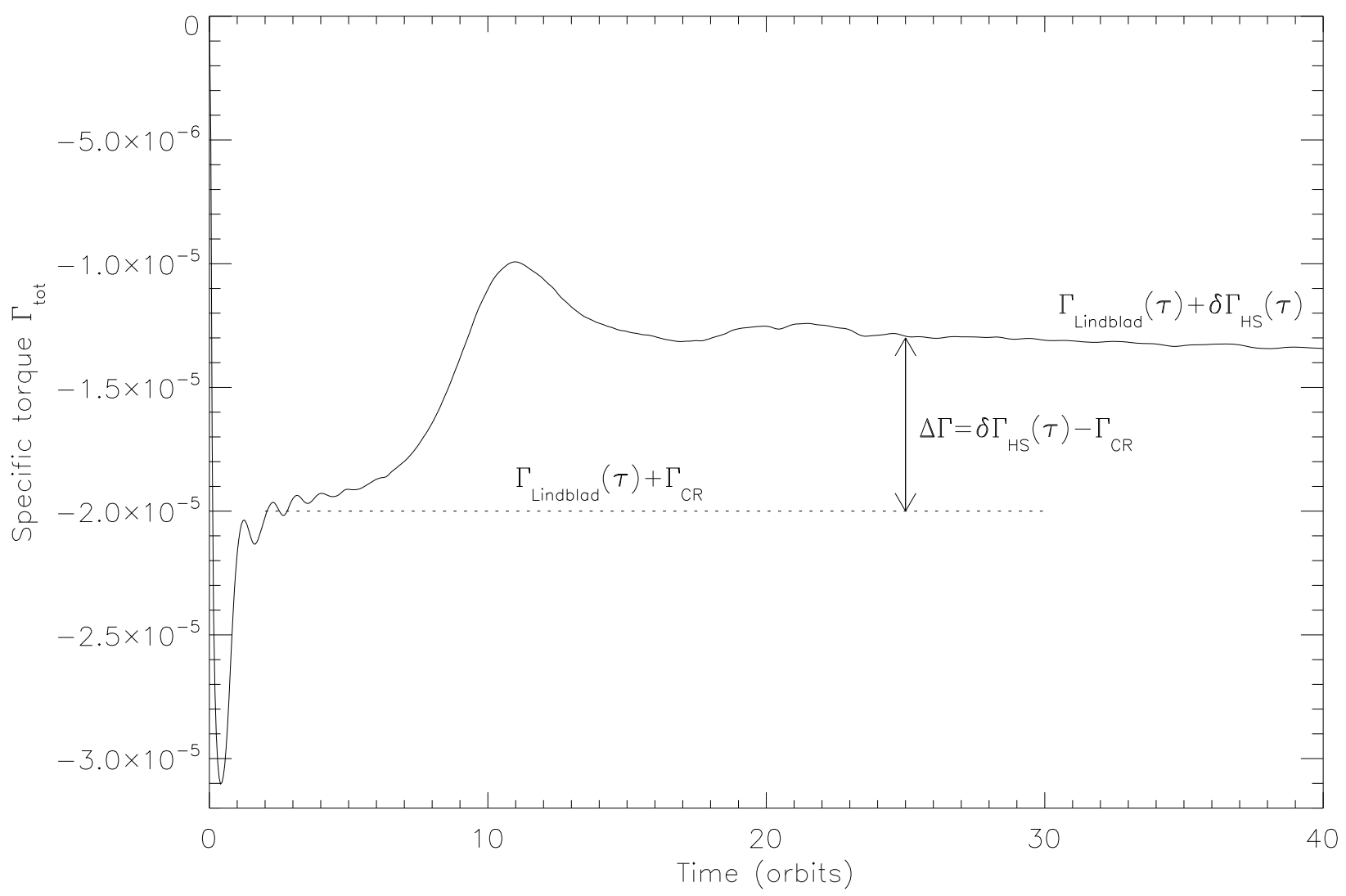

Fig. 7.- Measurement of $\Delta \Gamma$. For a given run (here, $\tau=-1.35, \epsilon=0.3$ ), we monitor the total torque $\Gamma_{\text {tot }}$ as a function of time. The differential Lindblad torque $\Gamma_{\text {Lindblad }}$ and the linear co-rotation torque $\Gamma_{\mathrm{CR}}$ only take a few orbits to establish, then the horseshoe drag develops, taking place of the linear corotation torque (Paardekooper \& Papaloizou 2009b). We measure $\Delta \Gamma=\delta \Gamma_{\mathrm{HS}}-\Gamma_{\mathrm{CR}}$, which, as $\Gamma_{\mathrm{CR}}$ does not depends on $\tau$, should trace the dependency of the horseshoe drag on $\tau$. The values of $\Gamma_{\text {Lindblad }}+\Gamma_{\mathrm{CR}}$ and $\Gamma_{\text {Lindblad }}+\delta \Gamma_{\mathrm{HS}}$ are measured by averaging $\Gamma_{\text {tot }}$ between 2 and 3 orbits on the one hand, and between 30 and 35 on the other hand. Slight changes of theses values did not bring differences. 
$\epsilon=0.3$ for $|\tau| \sim 1$, which seem to disappear for the larger value of $\epsilon$. This is linked to the appearance of a "trapped region", which we explain in the next section.

\subsection{A note on the softening length}

The streamlines in the inner part of the horseshoe region, near the planet, exhibit different behaviors as a function of the softening length, as shown in figure 9. We can distinguish two cases:

- a case where there are three stagnation points, as discussed in Masset et al. (2006a), with almost the same radius $r_{c}$. The O-point is associated to the planet, being located almost at the same azimuth. One of the X-points lies on the separatrices. We call it the outer X-point. Similarly, the other X-point is the one we refer to as the inner X-point.

- a case where there is only one X-point, at the intersection of the separatrices.

One can continuously go from the first situation to the last one by varying the softening length. The inner X-point and the O-point merge at some point, yielding a one stagnation point configuration. A lower softening length favors the existence of an inner X-point, but it is not the only parameters that determines this property of the flow. The temperature gradient also plays a role (a smaller gradient favors the existence of a inner $\mathrm{X}$ point, while at large gradient we have a one stagnation point situation). We have seen this for two values of $\epsilon$, and present this for varying values of $\tau$ in figure 10. The existence of three stagnation points appears to be correlated with the discontinuities at $\tau= \pm 1$. We comment on this behavior in the next section. 


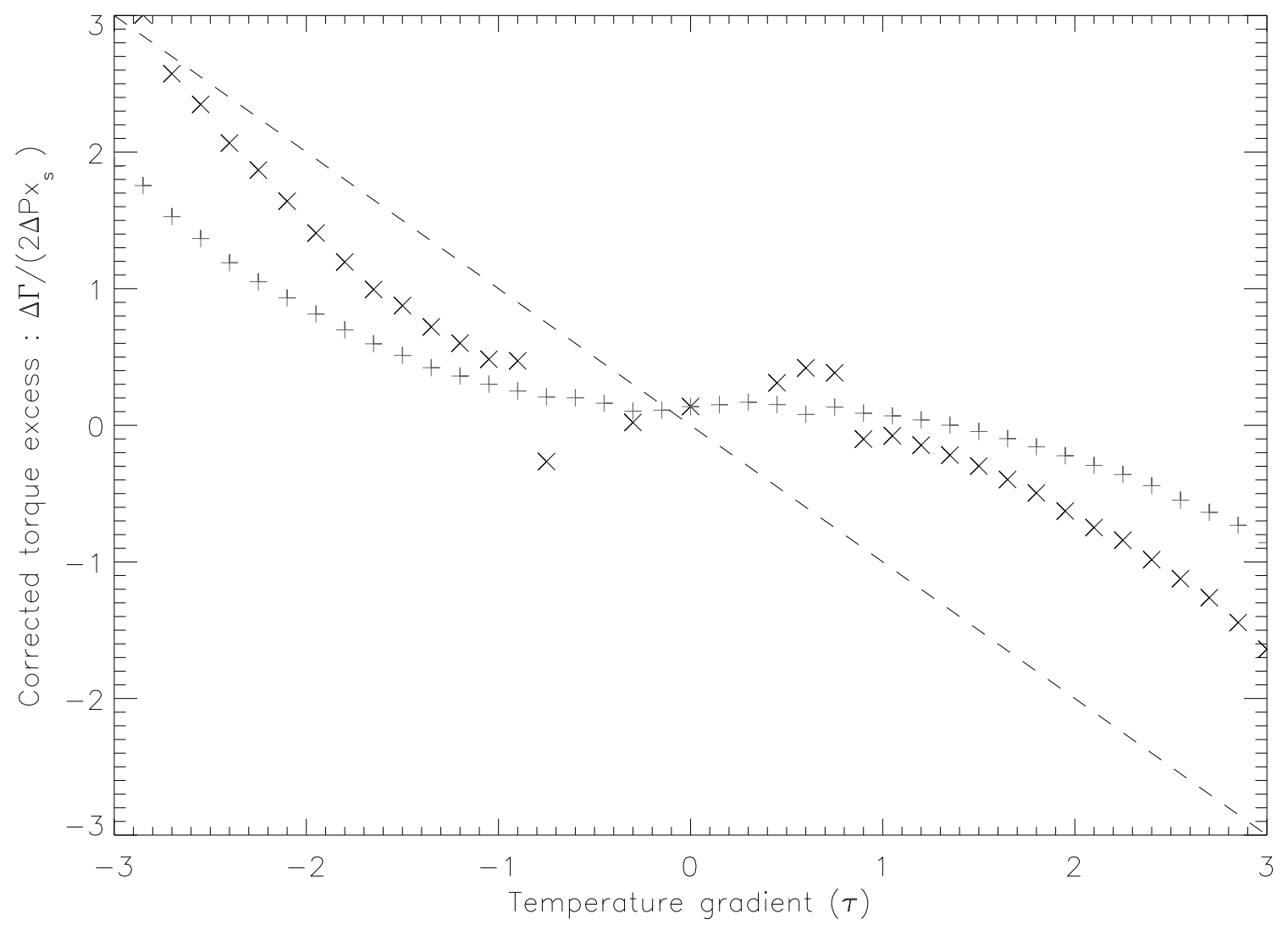

Fig. 8.- Non-isothermal torque excess. We plot this quantity as a function of $\tau$, for two sets of runs: $\epsilon=0.3(\mathrm{X})$, and $\epsilon=0.5(+)$. The dashed line represents our crude estimate of equation (59). A proper linear regression fit of the simulated points would yield a slope within $\pm 25 \%$ of our prediction. The break of continuity that we see for $\epsilon=0.3$ exists also for $\epsilon=0.5$, but is much less apparent, as it takes place at $\tau \approx \pm 0.5$, and results in a much smaller jump of the corrected torque excess. 


\subsection{The different regimes in the locally isothermal case}

The production of vortensity takes place near the planet, during the horseshoe U-turns, where the gradients of pressure (dominated by the planet's potential) and of temperature are not aligned. For the sake of definiteness, we assume that there is a negative temperature gradient. Therefore a negative amount of vortensity is produced behind the planet and a positive amount of vortensity is produced in front of it. In an idealized situation with a unique X-point centered on the planet, the negative perturbed vortensity is fully advected to the rear of the planet, while the positive perturbed vortensity perturbation is advected towards positive azimuth. They respectively yield a positive surface density perturbation behind the planet (for the reasons exposed in section 3.4, which are valid azimuthally far from the planet, where the gradients of temperature and density are aligned) and a negative perturbation of surface density in front of the planet (hence a negative non-isothermal torque excess).

In a realistic situation, however, the fact that the $\mathrm{X}$-point is not centered on the planet changes this picture. We take the example of $\tau \sim-1$ (one can refer to figure 9b to see a streamline map that looks similar to this situation). The material at the rear of the $\mathrm{X}$ point is advected to the rear of the planet, as expected. But some of the material (and the resulting underlying created positive vortensity) at the front of the $\mathrm{X}$ point, but still at the rear of the planet, is eventually advected to the front of the planet. This lowers the torque excess exerted on the planet, as a part of the positive vortensity created at the rear of the planet and advected to the front cancels out with the negative vortensity created and advected to the front. This explains why the simulated points in figure 8 are below our prediction for $\tau<-1$.

As $|\tau|$ decreases, a "trapped region" appears, enclosed between the two X-points, and centered on the O-point. The material in this region orbits around the planet, and does 
not contribute to the torque. In fact, the created vortensity supported by this material is trapped here, and even eventually flattens out (under the action of phase mixing, which requires very long times). For $\tau<0$, the azimuth of the $\mathrm{O}$-point is positive, and the material trapped in this region essentially has a negative created vortensity. Hence, when the trapped region appears, a significant part of the advected negative vortensity disappears from the torque, resulting in a smaller torque excess. This likely explains the discontinuity in figure 8 at $\tau \simeq-1$.

Similar arguments hold for the case $\tau>0$ (we then have to change the sign of the vortensity produced, as well as the sign of the azimuth of the outer and inner X-points, as can be seen in figure [10).

For $|\tau|>1, \Delta \Gamma$ decreases with $\tau$ as announced, with a slope that is roughly the one predicted in section 6.1, For $|\tau|<1$, however, this slope is reverted. The reason for this behavior is unclear, and its study requires an in-depth streamline analysis, as well as a quantitative estimate of the vortensity produced in the vicinity of the separatrices, which is beyond the scope of this qualitative description.

\section{Conclusion}

We have derived an expression for the horseshoe drag that takes into account the effects of pressure. It yields a result identical to previous estimates of the horseshoe drag, except that it explicitly takes into account the material beyond the horseshoe separatrices. The horseshoe drag therefore accounts for the torque arising from the whole corotation region, and therefore should deserve to be called corotation torque. We find that the horseshoe region is asymmetric, in a manner that depends on the vortensity gradient. Specifically, the part in front of the planet is wider for a decreasing vortensity profile in the disk, while the 
part behind the planet is more narrow. This asymmetry can be interpreted as an effect of evanescent pressure waves, launched from the downstream part of the horseshoe U-turns, which perturb the upstream region. Using a Bernoulli invariant, we derived this asymmetry for a globally isothermal disk, and found it to scale with the vortensity gradient and the disk's aspect ratio.

The impact of the evanescent waves on the horseshoe width can easily be interpreted as a consequence of an azimuthal pressure gradient, whose torque counteracts or adds to the torque required to perform a U-turn, resulting either in a wider or in a narrower horseshoe region.

This asymmetry does not alter the standard horseshoe drag. Basically, the supplementary torque excess resulting from the widening of one side of the horseshoe region is balanced by the torque deficit resulting of the shrinking of the other side of the horseshoe region.

As the concept of horseshoe drag has proved of increasing importance for planetary migration in a number of recent works (Masset et al. 2006b.a; Paardekooper \& Mellema 2006; Baruteau \& Masset 2008; Paardekooper \& Papaloizou 2008, 2009a,.b; Kley \& Crida 2008), it is crucial to adopt a proper estimate of the correct horseshoe width when evaluating the corresponding horseshoe drag. We find that the correct mean width of the horseshoe region should be estimated as given by equation (44). As the asymmetry is relatively mild for typical vortensity gradients and aspect ratios, a simple arithmetic mean of the front and rear widths also yields estimates that are sufficiently accurate.

Our analysis is based on the use of a Bernoulli invariant, which is made possible by the hypothesis that the disk is globally isothermal, hence barotropic. Such an approach is not possible for a locally isothermal disk. In such a situation, vortensity is created near the planet, which gives rise to a supplementary torque. We have presented a qualitative study 
of this supplementary torque, whose dependence on the temperature gradient appears to be intimately linked to the topology of the flow in the planet vicinity, and which exists in a disk even without a vortensity gradient. We also provide a crude estimate of this dependence, which is meant to apply to situations with only one stagnation point (i.e. at large temperature gradient). We stress that this supplementary torque is not strong enough to reverse planetary migration in a disk with a surface density that decreases outwards as a power law of radius, even with unrealistic temperature gradients. Nevertheless, it should be considered whenever accurate estimates of the migration rate are required, as its amplitude can amount to about one third of the total horseshoe drag. A systematic study of these non-isothermal effects, and their generalization to the three-dimensional case, should be undertaken in order to provide reliable estimates of this supplementary torque, that we have called the non-isothermal torque excess.

An important feature of this excess is that it is linked to an edge effect of the horseshoe drag. The bulk horseshoe drag, which corresponds to the classical expression, always scales with the vortensity gradient. Any dependence on another parameter of the disk (such as the temperature gradient) manifests itself as evanescent waves excited at the downstream separatrices, where they yield a vortensity sheet as the most tangible imprint on the flow. In the case of a locally isothermal disk, the lack of an invariant and the strong dependence on the topology of the flow render the situation very complex and hardly tractable. In the case of an adiabatic flow, one can exhibit, under certain circumstances, an invariant of the flow. This allows a rigorous analysis of the horseshoe drag and of the main properties of the horseshoe region. This analysis is presented in paper II.

The numerical simulations performed in this work have been run on the 92 core cluster funded by the program Origine des Planètes et de la Vie of the French Institut National des Sciences de l'Univers. Partial support from the COAST project (COmputational 
ASTrophysics) of the CEA is also acknowledged. The authors also wish to thank G.

Koenigsberger for hospitality at the Instituto de Ciencias Fisicas of UNAM, Mexico, and acknowledge partial support from CONACYT project number 24936. 

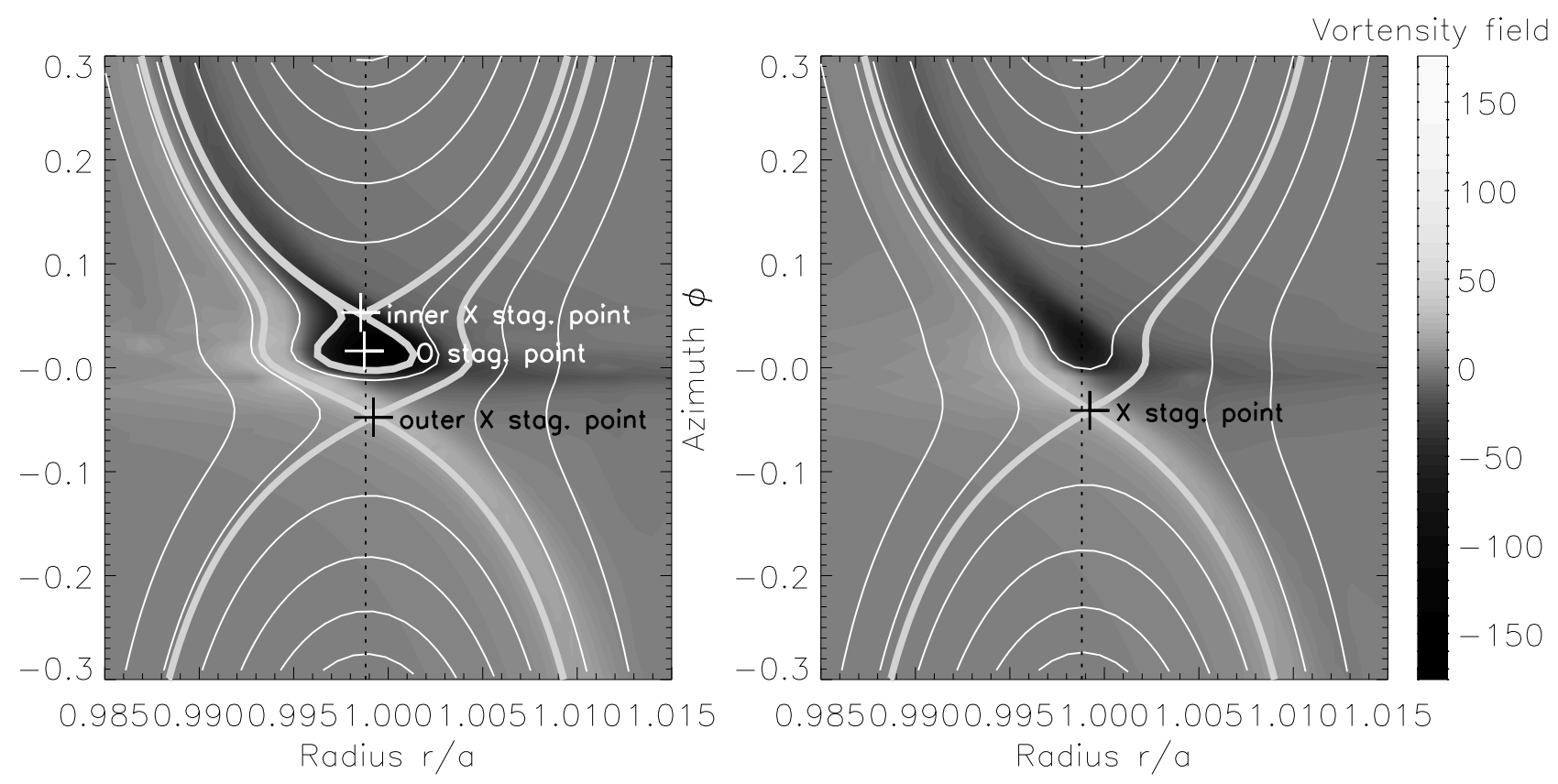

Fig. 9.- Appearance of a trapped region. This is a close overview of the horseshoe region, at $\mathrm{T}=13$ orbits, close to the planet, which is at located at $\phi=0$ and $r=1$. The temperature gradient is $\tau=-0.9$. The left panel show the $\epsilon=0.3$ case, while the right panel shows the $\epsilon=0.5$ case. The thin dotted line represents the corotation. The underlying field is the perturbed vortensity. The thin white lines are the streamlines, and the bold ones represents the separatrices. We clearly see the advection of the vortensity created, and the advection of negative and positive vortensity to the front region (since, here, the outer stagnation point is behind the planet). When the inner stagnation point disappears, the material executing a U-turn in front of the planet passes faster by the planet, hence a smaller creation of negative vortensity (hardly seen with the grey scale, but there is a difference of about one half), while the creation of positive vortensity behind the planet remains of the same order. 


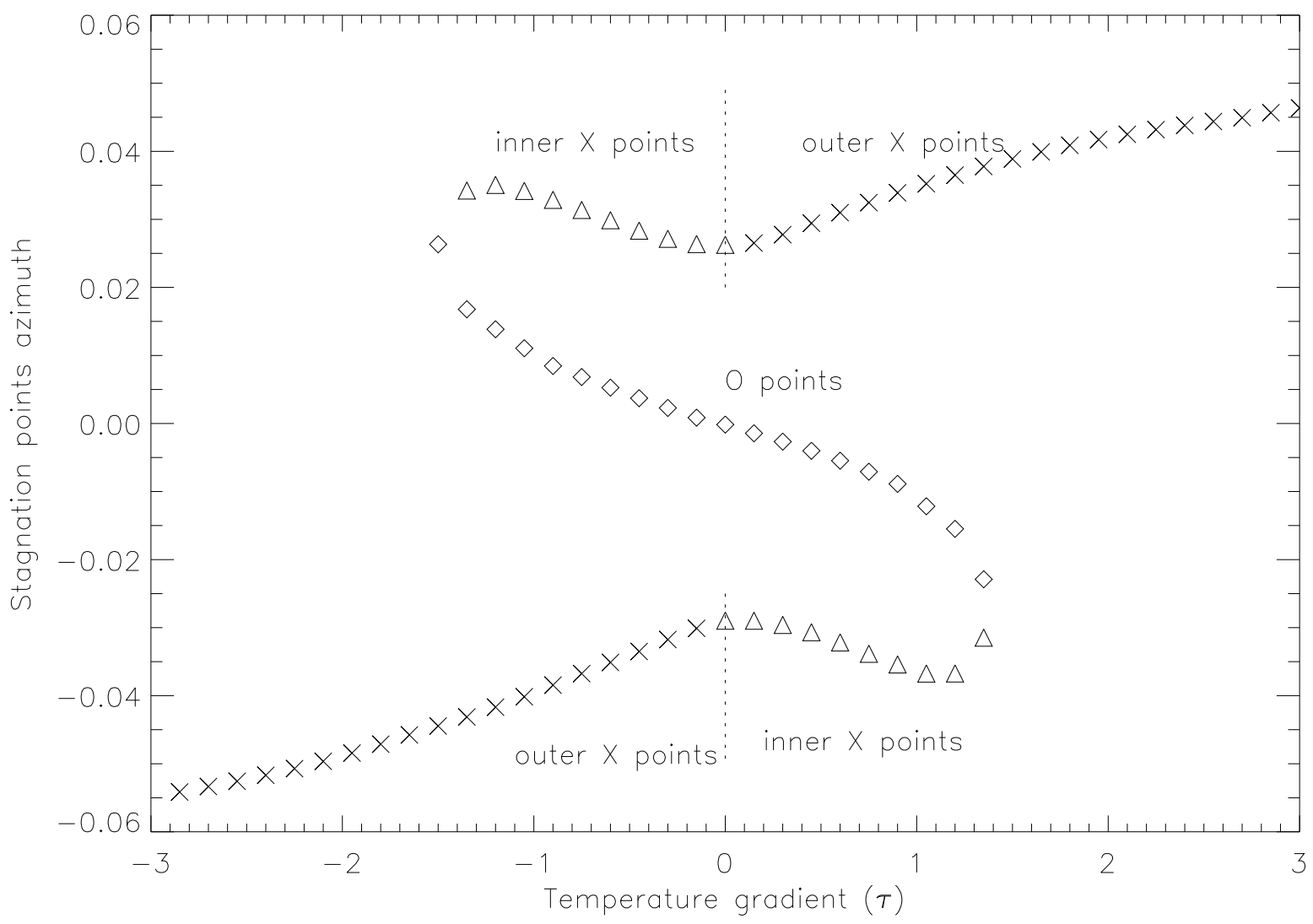

Fig. 10.- Azimuth of the stagnation point(s) as a function of the temperature gradient, at $\mathrm{T}=5$ orbits. The variation of the radial position is much smaller, although it exhibits a reproducible behavior (the front (rear) X stagnation point being always at $r<a(r>a)$ ). For $|\tau|<1.5$, there is one $\mathrm{O}$ point, and two X-points, as indicated on the figure. At larger times, the inner X-point and the O-point tend to merge, reducing the interval upon which there are three stagnation points. Nevertheless, it remains quite large $(|\tau|<1)$ in steady state. 


\section{REFERENCES}

Baruteau, C., \& Masset, F. 2008, ApJ, 672, 1054

Goldreich, P., \& Tremaine, S. 1979, ApJ, 233, 857

-. 1980, ApJ, 241, 425

Kley, W., \& Crida, A. 2008, A\&A, 487, L9

Korycansky, D. G., \& Pollack, J. B. 1993, Icarus, 102, 150

Masset, F. 2000a, A\&AS, 141, 165

Masset, F. S. 2000b, in Astronomical Society of the Pacific Conference Series, Vol. 219, Disks, Planetesimals, and Planets, ed. G. Garzón, C. Eiroa, D. de Winter, \& T. J. Mahoney, 75-+

Masset, F. S. 2001, ApJ, 558, 453

-. 2002, A\&A, 387, 605

Masset, F. S., D’Angelo, G., \& Kley, W. 2006a, ApJ, 652, 730

Masset, F. S., Morbidelli, A., Crida, A., \& Ferreira, J. 2006b, ApJ, 642, 478

Masset, F. S., \& Papaloizou, J. C. B. 2003, ApJ, 588, 494

Paardekooper, S. ., \& Papaloizou, J. C. B. 2009a, MNRAS, 394, 2297

Paardekooper, S.-J., \& Mellema, G. 2006, A\&A, 459, L17

Paardekooper, S.-J., \& Papaloizou, J. C. B. 2008, A\&A, 485, 877

Paardekooper, S. J., \& Papaloizou, J. C. B. 2009b, MNRAS, 394, 2283 
Tanaka, H., Takeuchi, T., \& Ward, W. R. 2002, ApJ, 565, 1257

Ward, W. R. 1986, Icarus, 67, 164

-. 1988, Icarus, 73,330

-. 1989, ApJ, 336, 526

Ward, W. R. 1991, in Lunar and Planetary Institute Conference Abstracts, 1463-+

Ward, W. R. 1992, in Lunar and Planetary Institute Conference Abstracts, 1491-+ 